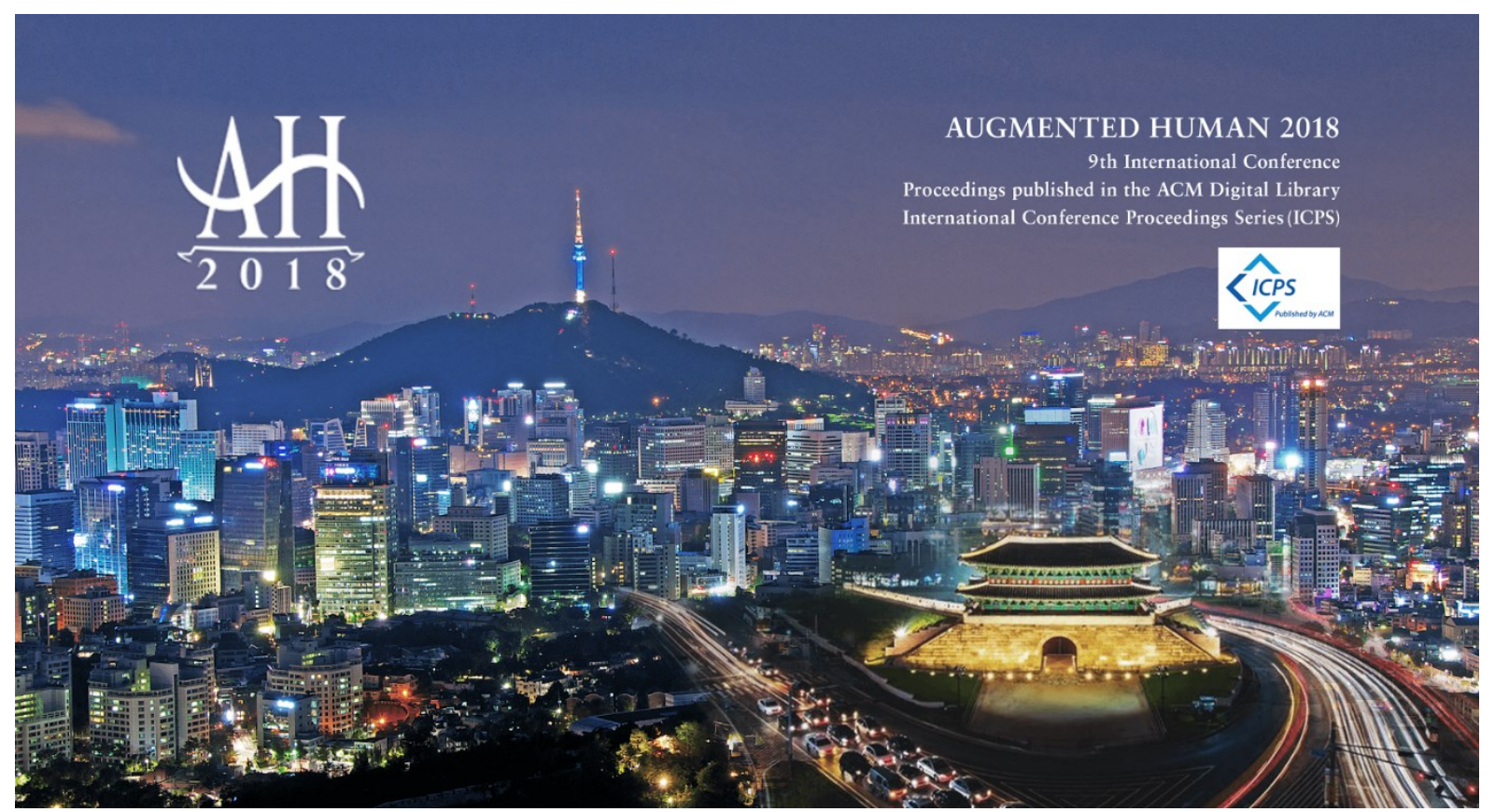

Hosted by
8 The HCI Society of Korea $\&$ HCIK sig10+

Organized by 


\section{Proceedings of the 9th Augmented Human International Conference}

Seoul, Korea, Feb. 7-9 2018

AH2018

Program Co-Chairs:

Jean-Marc Seigneur, University of Geneva, Switzerland

Gerard Jounghyun Kim, Korea University, Korea

General Co-Chairs:

Woontack Woo, KAIST, Korea

Juhyun Eune, Seoul National University, Korea

Sponsors:

Korea Advanced Institute of Science and Technology (KAIST)

Seoul National University

ACM International Conference Proceedings Series

ACM Press 


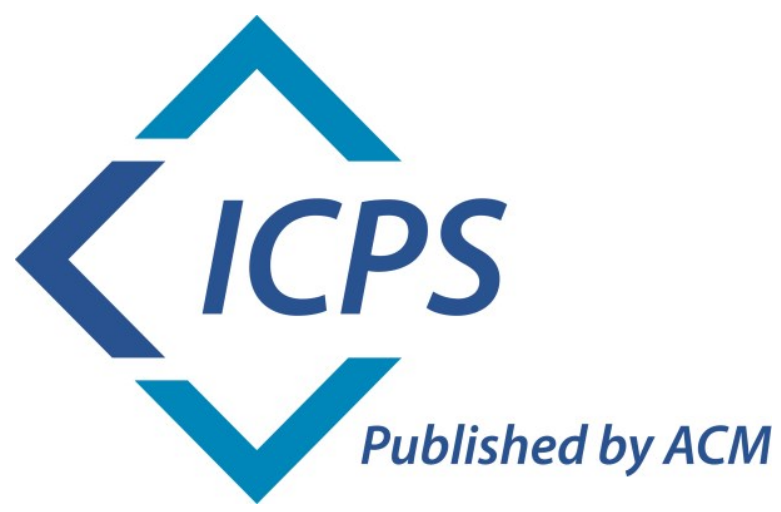

The Association for Computing Machinery

2 Penn Plaza, Suite 701

New York New York 10121-0701

ACM COPYRIGHT NOTICE. Copyright $\odot 2018$ by the Association for Computing Machinery, Inc. Permission to make digital or hard copies of part or all of this work for personal or classroom use is granted without fee provided that copies are not made or distributed for profit or commercial advantage and that copies bear this notice and the full citation on the first page. Copyrights for components of this work owned by others than ACM must be honored. Abstracting with credit is permitted. To copy otherwise, to republish, to post on servers, or to redistribute to lists, requires prior specific permission and/or a fee. Request permissions from Publications Dept., ACM, Inc., fax +1 (212) 869-0481, or permissions@acm.org.

For other copying of articles that carry a code at the bottom of the first or last page, copying is permitted provided that the per-copy fee indicated in the code is paid through the Copyright Clearance Center, 222 Rosewood Drive, Danvers, MA 01923, +1-978-750-8400, +1-978-750-4470 (fax).

Notice to Past Authors of ACM-Published Articles

ACM intends to create a complete electronic archive of all articles and/or other material previously published by ACM. If you have written a work that was previously published by ACM in any journal or conference proceedings prior to 1978, or any SIG Newsletter at any time, and you do NOT want this work to appear in the ACM Digital Library, please inform permissions@acm.org, stating the title of the work, the author(s), and where and when published.

ACM ISBN: 978-1-4503-5415-8 


\section{Introduction}

The 9th Augmented Human International Conference (AH2018) has gathered scientific papers from many different disciplines. Many papers concentrated on building the human augmentation experience (AX), which was the chosen focus theme of the event. Unfortunately, few papers investigated the ethical or safety issues of augmented human technologies. The next edition may bring more papers on this essential aspect that must be taken into account for a long-term success of these technologies. Check http://www.augmented-human.com to subscribe to the mailing list and to be informed about the next editions of the conference. Consider also checking the Springer Journal on Augmented Human Research to get extended research papers on the topic!

\section{Acknowledgments}

The AH research community is truly multidisciplinary and it is a challenge to gather reliable expert scientific reviewers in so many fields. Therefore, we thank a lot all the co-chairs, program committee members, reviewers and subreviewers who have sent their reviews on time. We also thank the ACM for publishing the proceedings of the conference in its online library.

\section{Program Co-Chairs:}

Jean-Marc Seigneur, University of Geneva, Switzerland

Gerard Jounghyun Kim, Korea University, Korea

General Co-Chairs:

Woontack Woo, KAIST, Korea

Juhyun Eune, Seoul National University, Korea

\section{Poster Chair:}

Dongsik Jo, ETRI, Korea

\section{Demo Chairs:}

Jeakweon Han, Hanyang University, Korea

Hyungseok Kim, Konkuk University, Korea

\section{Publication Chair:}

Geehyuk Lee, KAIST, Korea

\section{Logistic / Social Chair:}

Jeongyun Heo, Kookmin University, Korea

\section{Publicity Chair:}

Kwangsu Cho, Yonsei University, Korea

Finance \& Registration Chair:

Junho Choi, Yonsei University, Korea 


\section{Industry Chair:}

Jihoon Jeong, Kyung Hee Cyber University, Korea

\section{Student Volunteer Chair:}

Hyung-il Kim, KAIST, Korea

\section{Program Review Committee:}

Florian Alt, Ludwig Maximilian University of Munich Andreas Bulling, Max Planck Institute for Informatics Maurizio Caon, University of Applied Sciences of Western Switzerland of Fribourg Bing-Yu Chen, National Taiwan University

Kwangsu Cho, Yonsei University

Junho Choi, Yonsei University Ashley Colley, University of Lapland Ellen Yi-Luen Do, Georgia Institute of Technology Jaroslaw Domaszewicz, Warsaw University of Technology Juhyun Eune, Seoul National University Masaaki Fukumoto, NTT DoCoMo Research Labs.

Patricia Gouveia, Universidade de Lisboa Axel Graeser, University Bremen Cathal Gurrin, Dublin City University Jeakweon Han, Hanyang University Jeongyun Heo, Kookmin University Christian Holz, Hasso Plattner Institute Jochen Huber, Synaptics Emin Huseynov, University of Geneva Jane Hwang, Korea Institute of Science and Technology Masahiko Inami, Keio University

Yuta Itoh, Technical University of Munich Seigneur Jean-Marc, University of Geneva Seokhee Jeon, Kyung Hee University Jihoon Jeong, Kyung Hee Cyber University Dongsik Jo, ETRI Gerard Jounghyun Kim, Korea Universtiy Howon Kim, ETRI Hyungseok Kim, Konkuk University Namgyu Kim, Dong-Eui University Hideki Koike, University of Tokyo

Yuichi Kurita, Hiroshima University Geehyuk Lee, KAIST

Jongwon Lee, Sejong University

Joohaeng Lee, ETRI

Fabien Lotte, INRIA

Kris Luyten, Hasselt University

Shachar Maidenbaum, HUJI

Paul McCullagh, Ulster University 
René Meier, Lucerne University of Applied Sciences

Guillaume Moreau, Ecole Centrale de Nantes - CERMA

Joel Murphy, openBCI

Suranga Nanayakkara, Singapore University of Technology and Design

Anton Nijholt, University of Twente

Takuya Nojima, University of Electro-Communications

Jean-Marie Normand, Ecole Centrale de Nantes

Ian Oakley, University of Madeira

Yoichi Ochiai, Universitty of Tsukuba

Jongil Park, Hanyang Univeristy

Jun Park, Hongik University

Isabel Pedersen, University of Ontario Institute of Technology

Martin Rieser, De Montfort University

Enrico Rukzio, Ulm University

Hideo Saito, Keio University

Gionata Salvietti, University of Siena

Johannes Schoening, University of Bremen

Roy Shilkrot, Stony Brook University

Redha Taiar, University of Reims

Kristof Van Laerhoven, University of Siegen

Athanasios Vourvopoulos, Madeira-ITI, Universidade da Madeira

Daniel Wessolek, Futurium gGmbH

Raphael Wimmer, University of Regensburg

Anusha Withana, Singapore University of Technology and Design

Woontack Woo, KAIST

\section{Subreviewers:}

Junsung Bang, Florian Bockes, Michael Braun, Jacky Casas, Angel Mario Castro Martinez, Samantha Chan, Jagmohan Chauhan, Yi-Ling Chen, Karl Daher, Thomas Dreja, Joël Dumoulin, Tim Dünte, Don Samitha Elvitigala, Henrique Galvan Debarba, Chiara Gaudeni, Jerônimo Grandi, Jürgen Hahn, Satoshi Hashizume, Teresa Hirzle, Takayuki Hoshi, Yining Hu, Axel Hösl, Nicholas Katzakis, Seong-Woo Kim, Sung Woo Kim, Naoya Koizumi, Morten Kolbæk, Nojun Kwak, Huy Viet Le, Dongjun Lee, Christian Mai, Sven Mayer, Leonardo Meli, Jelena Mladenovic, Preetha Moorthy, Naoya Muramatsu, Philipp Matthias Müller, Aditya Nittala, Kazuki Otato, Gianluca Paolocci, Roshan Lalintha Peiris, Max Pfeiffer, Ken Pfeuffer, Tu-Hoa Pham, Huy Phan, Marko Radeta, Simon Ruffieux, Samanta Scala, Adwait Sharma, Mohamed Soliman, Priyashri Sridhar, Julian Steil, Yuta Sugiura, Kazuki Takazawa, Benjamin Tannert, Hsin-Ruey Tsai, Yuji Uema, Vivek Venugopalan, Hsiang-Yun Wu, Shi Yilei, Kening Zhu 


\section{Table of Contents}

\section{Full Papers}

Article 1: “Air Mounted Eyepiece: Optical See-Through HMD Design with Aerial Optical”, Kazuki Otao, Yuta Itoh, Kazuki Takazawa, Hiroyuki Osone, and Yoichi Ochiai.

Article 2: "Investigation of Tracer Particles Realizing 3-Dimensional Water Flow Measurement for Augmented Swimming Training”, Shogo Yamashita, Shunichi Suwa, Takashi Miyaki, and Jun Rekimoto.

Article 3: "Couples Designing their Living Room Together: a Study with Collaborative Handheld Augmented Reality”, Joon Gi Shin, Gary Ng, and Daniel Saakes.

Article 4: "Augmenting Memory Recall in Work Meetings: Establishing a Quantifiable Baseline", Evangelos Niforatos, Matías Laporte, Agon Bexheti, and Marc Langhein-rich.

Article 5: "Virtual Participation in Ukiyo-e Appreciation using Body Motion", Shota Kusajima, Takuya Takahashi, and Yasuyuki Sumi.

Article 6: "AR Timewarping: A Temporal Synchronization Framework for Real-Time Sensor Fusion in Head-Mounted Displays”, Peter Kim, Jason Orlosky, and Kiyoshi Kiyokawa.

Article 7: "Telewheelchair: the Remote Controllable Electric Wheelchair System combined Human and Machine Intelligence", Satoshi Hashizume, Ippei Suzuki, Kazuki Takazawa, Ryuichiro Sasaki, and Yoichi Ochiai.

Article 8: "Command Selection in Gaze-based See-through Virtual Image-Guided Environments", Hoorieh Afkari, David Gil de Gómez Pérez, and Roman Bednarik.

Article 9: "HIVE Tracker: a tiny, low-cost, and scalable device for sub-millimetric 3D positioning”, Darío R. Quiñones, Gonçalo Lopes, Danbee Kim, Cédric Honnet, David Moratal, and Adam Kampff.

Article 10: "A Motion Recognition Method Using Foot Pressure Sensors", Ayumi Ohnishi, Tsutomu Terada, and Masahiko Tsukamoto.

Article 11: "Design and Study of a Multi-Channel Electrical Muscle Stimulation Toolkit for Human Augmentation”, Michinari Kono, Yoshio Ishiguro, Takashi Miyaki, and Jun Rekimoto.

Article 12: "Sync Class: Visualization System for In-Class Student Synchronization", Katsuya Fujii, Plivelic Marian, Dav Clark, Yoshi Okamoto, and Jun Rekimoto.

Article 13: “AVATAREX: Telexistence System based on Virtual Avatars”, Timo Koskela, Mounib Mazouzi, Paula Alavesa, Minna Pakanen, Ilya Minyaev, Eero Paavola, and Jere Tuliniemi

Article 14: “V8 Storming: How Far Should Two Ideas Be?”, Yui Kita, and Jun Rekimoto.

Article 15: "Seeing is Smelling: Localizing Odor-Related Objects in Images", Sangyun Kim, Junseok Park, Junseong Bang, and Haeryong Lee.

Article 16: "GlassPass: Tapping Gestures to Unlock Smart Glasses”, MD. Rasel Islam, Doyoung Lee, Liza Suraiya Jahan, and Ian Oakley. 


\section{Short Papers}

Article 17: "Open Palm Menu: A Virtual Menu Placed in Front of the Palm”, Takumi Azai, Mai Otsuki, Fumihisa Shibata, and Asako Kimura.

Article 18: "Recognition and Feedback of Vowel Utterance with a Good Mouth Shape Based on Sensing Platysma Muscle Bulging,, Yukihiro Nishimura and Tomoko Hashida.

Article 19: "Wanding Through Space: Interactive Calibration for Electric Muscle Stimulation”, Henning Pohl, Kasper Hornbæk, and Jarrod Knibbe.

Article 20: "GoalBaural: A Training Application for Goalball-related Aural Sense", Takahiro Miura, Shimpei Soga, Masaki Matsuo, Masatsugu Sakajiri, JunjiOnishi, and Tsukasa Ono.

Article 21: “An Interactive 4D Vision Augmentation of Rapid Motion”, Tao Tao, Photchara Ratsamee, Yuki Uranishi, Kiyoshi Kiyokawa, TomohiroMashita, and Haruo Takemura.

Article 22: "A Detachable Exoskeleton Inter-face that Duplicates the User's Hand Posture and Motions", Genki Toyama and Tomoko Hashida.

Article 23: "FaceRubbing: Input Technique by Rubbing Face using Optical Sensors on Smart Eyewear forFacial Expression Recognition”, Katsutoshi Masai, Yuta Sugiura, and Maki Sugimoto.

Article 24: “A soft exoskeleton suit to reduce muscle fatigue with pneumatic artificial muscles”, Kosuke Tsuneyasu, Ayumu Ohno, Yoshiyuki Fukuda, KazunoriOgawa, Toshio Tsuji, and Yuichi Kurita.

Article 25: "Object-wise3D Gaze Mapping in Physical Workspace", Kakeru Hagihara, Keiichiro Taniguchi, Irshad Abibouraguimane, Yuta Itoh,Keita Higuchi, Jiu Otsuka, Maki Sugimoto, and Yoichi Sato.

\section{Poster Papers}

Article 26: "Enhanced Pressure-Based Multimodal Immersive Experiences", Taeyong Kim and Jeremy R. Cooperstock.

Article 27: "VR Planning Toolkit to Simulate Physical and Virtual Configurations: A Case Study of an Indoor VR Roller Coaster Augmenting Experience”, Dongsik Jo, Yongwan Kim, Woojin Jeon, Yongsun Kim, Hongki Kim, Ki-Hong Kim, and Seungho Kwak.

Article 28: "Augmenting Smart Object Interactions with Smart Audi”, Jing Yang and Gábor Sörös.

Article 29: "Towards Enhancing Emotional Responses to Media using Auto-Calibrating Electric Muscle Stimulation (EMS)”, Takashi Goto, Benjamin Tag, Kai Kunze, and Tilman Dingler.

Article 30: "Reinforced Suit Using Low Pressure Driven Artificial Muscles For Baseball Bat Swing", Watura Sakoda, Antonio Vega Ramirez, Kazunori Ogawa, Toshio Tsuji, and Yuichi Kurita.

Article 31: "Visual Field Visualizer: Easier \& Scalable way to be Aware of the Visual Field", Ngoc Thi Nguyen, Suranga Nanayakkara, and Hyowon Lee.

Article 32: "Exo-Balancer: Design Method of Personalized Stabilizers for Shooting Actions", Ryota Kawamura, Kazuki Takazawa, Riku Iwasaki, Kenta Yamamoto, and Yoichi Ochiai.

Article 33: "Restorative Effects of Exercise in Virtual Environments", Taekyu Kim and Sangwon Lee. 
Article 34: "TouchSense: Classifying and Measuring the Force of Finger Touches with an Electromyography Armband”, Vincent Becker, Pietro Oldrati, Liliana Barrios, and Gábor Sörös.

Article 35: "Paralogue: A Remote Conversation System Using a Hand Avatar which Postures are Controlled with Electrical Muscle Stimulation”, Shin Hanagata and Yasuaki Kakehi.

\section{Demo Papers}

Article 36: "MOYA: Interactive AI toy for children to develop their language skills", Ji Yoon Ahn, Dong Wan Kim, Yong Hyeon Lee, Woori Kim, Jeong Kuk Hong, Yeonbo Shim, Jin Ho Kim, Juhyun Eune, and Seong-Woo Kim.

Article 37: "e-mmersive Book: the ARbook that assists the syntopical reading", Shinhyo Kim, Jihyun Park, and Jusub Kim.

Article 38: "Unconstrained Neck: Omnidirectional Observation from an Extra Robotic Neck", Lichao Shen, MHD Yamen Saraji, Kai Kunze, and Kouta Minamizawa.

Article 39: "Distributed Metaverse: Creating Decentralized Blockchain-based Model for Peer-to-peer Sharing of Virtual Spaces for Mixed Reality Applications”, Bektur Ryskeldiev, Yoichi Ochiai, Michael Cohen, and Jens Herder.

Article 40: "Introducing Smart Pillow Using Actuator Mechanism, Pressure Sensors, and Deep LearningBased ASR”, Seung Hee Yang, Sangwoo Park, Taemyung Yang, Ilhyung Jin, Wooil Kim,Chingwei Liu, Seong-Woo Kim, and Juhyun Eune.

Article 41: “Augmented Memory: Site-Specific Social Media with AR”, Seonghun Park and Jusub Kim.

Article 42: "SmartFiber: Reconfigurable Shape Changing Interface", Masaru Ohkubo and Takuya Nojima.

Article 43: "Automated Data Gathering and Training Tool for Personalized "Itchy Nose"”, Juyoung Lee, Hui-Shyong Yeo, Thad Starner, Aaron Quigley, Kai Kunze, and Woontack Woo.

Article 44: "ToolShaker: Presen-tation Technique for "as-is" Display of Daily Commodities", Hayato Dogai, Maho Oki, and Koji Tsukada. 